\title{
Bronchial stenosis and sclerosing mediastinitis: an uncommon complication of external thoracic radiotherapy
}

\author{
St. Dechambre*, J. Dorzee*, J. Fastrez**, C. Hanzen+, P. Van Houtte+, J.P. d'Odémont++
}

\begin{abstract}
Bronchial stenosis and sclerosing mediastinitis: an uncommon complication of external thoracic radiotherapy. St. Dechambre, J. Dorzee, J. Fastrez, C. Hanzen, P. Van Houtte, J.P. d'Odémont. CERS Journals Ltd 1998.

ABSTRACT: The side-effects of radiation therapy on the bronchial tree or on the mediastinum are seldom reported. In this setting, we report a case of sclerosing mediastinitis with bronchial stenosis discovered $1 \mathrm{yr}$ after external radiotherapy for lung cancer.
\end{abstract}

The patient was treated with a Dumont stent and has so far had an uneventful further course for up to $\mathbf{4 2}$ months. Bronchial stenosis related to mediastinal fibrosis after radiotherapy has not been reported previously.

Eur Respir J 1998; 11: 1188-1190.

\author{
Depts of *Radiology, **Thoracic Surgery \\ and ${ }^{++}$Respiratory Medicine, Centre Hos- \\ pitalier Régional Clinique Saint Joseph, \\ Mons, Belgium. +Dept of Radiotherapy, Clin- \\ ique Louis Caty, Baudour, Belgium. \\ Correspondence: J.P. d'Odemont \\ Dept of Respiratory Medicine \\ CHR Clinique Saint Joseph \\ 5 Avenue Baudouin de Constantinople \\ 7000 Mons \\ Belgium \\ Fax: 3265385599 \\ Keywords: Bronchial stenosis \\ sclerosing mediastinitis \\ thoracic radiotherapy \\ Received: June 231997 \\ Accepted after revision October 31997
}

Thoracic radiotherapy is one of the major treatment modalities in lung cancer. Radiation pneumonitis and fibrosis are well-known side-effects. "Radiation bronchitis" in the form of inflammatory reaction of the airways mucosa following therapeutic external-beam radiation and highdose endobronchial radiation (brachytherapy) has also been reported. Otherwise, there is very little mention in the literature regarding the effects of the radiation on the airways.

In this article, we report a case of sclerosing mediastinitis with bronchial stenosis discovered $1 \mathrm{yr}$ after external thoracic radiotherapy.

\section{Case report}

A right upper lobectomy for a peripheral adenocarcinoma of the ventral segment of the right upper lobe was performed on a $59 \mathrm{yr}$ old woman in November 1992. This patient had a relevant medical history of a 40 pack-year history of smoking, a left sylvian stroke in 1988, and an inferior myocardial infarction in 1990. The postoperative staging (tumour, node, metastasis (TNM) classification), using a systematic mediastinal sampling, was T3NOMO in view of an extension to the parietal pleura and the absence of metastases.

External radiation therapy was, therefore, performed using an $18 \mathrm{MeV}$ photon linear accelerator. Doses of 2 Gray (Gy) each were delivered in 27 fractions by a combination of anteroposterior parallel opposed fields (16 fractions), anterior direct fields (seven fractions), and anteroposterior opposed oblique angle wedge fields (four fractions). The total dose applied to the tumour bed and the right hilum was around 57 Gy. The total dose applied to the paratracheal left chain was approximately $45 \mathrm{~Gy}$, and the spinal cord received about $42 \mathrm{~Gy}$.

In November 1993, the patient complained of persistent cough and a gradual onset of dyspnoea. On physical examination, a decrease in the breath sounds over the right lung was noted, the left lung being clear; no other findings of note were detected. Blood results were normal except for a slight elevation of hepatic enzymes. The carcinoembryonic antigen (CEA) was $10 \mathrm{ng} \cdot \mathrm{mL}^{-1}$ (normal values $<7$ $\mathrm{ng} \cdot \mathrm{mL}^{-1}$ ), the level before the operation being $15 \mathrm{ng} \cdot \mathrm{mL}^{-1}$. The Mantoux test was negative.

Chest radiography disclosed a right hilar enlargement and a right apical thickening. Thoracic computed tomography (CT) showed the presence of a right paratracheal mass with extension around the right main-stem bronchus, the lumen of which was severely narrowed (fig. 1). The subcarinal area was also involved. A slight heterogeneous enhancement was observed after administration of contrast material. An area of soft tissue density containing dilated bronchi, with a straight edge corresponding to radiation fibrosis localized in the middle lobe with adjacent pleural thickening was present conforming to the radiation port. Magnetic resonance imaging (MRI) revealed that this mass had a low intensity signal both in T1 and $\mathrm{T} 2$ weighted images. This short $\mathrm{T} 2$ relaxation time was said to be suggestive of a benign fibrous process.

Fibreoptic examination revealed an $80 \%$ extrinsic stenosis of the right main-stem bronchus. This stenosis extended along the intermediate trunk. The mucosa had 


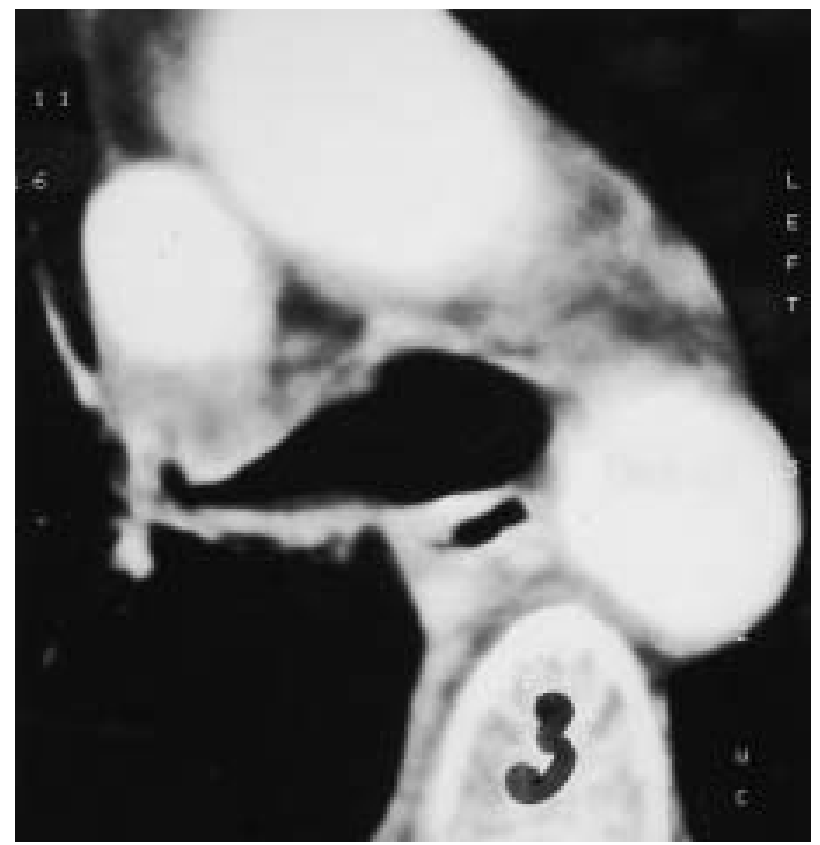

Fig. 1. - Computed tomography scan showing extrinsic narrowing of the right main-stem bronchus related to the presence of a right paratracheal and hilar mass.

a normal appearance. Multiple and extensive bronchial biopsies were unremarkable. Mediastinoscopy showed right peribronchial induration, with multiple adherences but without a distinct mass. In view of this and the tendency to bleed easily, biopsies were not performed. An endobronchial Dumont stent was positioned in the right main-stem bronchus in November 1993, resulting in a good clinical improvement.

Fibreoptic reassessment in July 1994 showed that the initial portion of the right main-stem bronchus and the distal portion of the trachea on the right side were distorted. A longer stent was therefore positioned to fit better with the stenosis. New bronchial biopsies performed at this time were unremarkable.

The patient was then regularly assessed by thoracic CT and bronchoscopy. Up to the present time, clinical, biological and imaging studies have been unsuccessful in determining any potential malignant process.

\section{Discussion}

Sclerosing mediastinitis is a rare condition characterized by an extensive fibrotic reaction creating a mediastinal or hilar mass, which may compress the tracheobronchial, digestive or vascular structures of the mediastinum. The most common cause of sclerosing mediastinitis is fungal infection, especially histoplasmosis, other main causes being tuberculosis (particularly in Europe), sarcoidosis, traumatic haemorrhage and drugs (methysergide). Association with autoimmune diseases, sclerosing cholangitis and Riedel's thyroiditis, is observed in some instances.

Sclerosing mediastinitis related to radiotherapy is rarely reported. Recently, in a series of 18 cases of sclerosing mediastinitis, Mole et al. [1] reported the case of three patients who received cytotoxic chemotherapy and exter- nal chest radiotherapy (radiation doses not specified) for a neoplastic disorder with lymph node involvement (two non-small cell carcinomas and one Hodgkin's disease). Whitcomb and SchwaRZ [2] described two cases of sclerosing mediastinitis, for which no apparent cause other than the external radiation therapy could be found. Irradiation was delivered with a total dose of $60 \mathrm{~Gy}$ for a lung squamous cell carcinoma with lymph node involvement, and with 40 Gy for a Hodgkin's disease. Similar observations were carried out on one single case of irradiated Hodgkin's disease both by RoDRIGUEZ GARCIA et al. [3] (with a radiation dose of $40 \mathrm{~Gy}$ ) and by Morrone et al. [4]. Bronchial stenosis, as seen in the present patient, was not rep-orted in these series.

In the setting of a history of malignancy in particular, reports highlight the difficulty of asserting the benign character of the sclerosing mediastinal process. Mediastinoscopy is currently performed $[1,5]$, given the well-known lack of accuracy of radiological explorations such as CT scanning and MRI. However, for the latter, decreased signal intensity in T2-weighted sequences may suggest that the mass is of a fibrotic nature [5].

Our presentation raises the issue of post-external radiation bronchial stenosis. Reports on direct adverse effects of radiotherapy on the tracheobronchial tree are scarce, most studies dealing with effects on the lung parenchyma, which is considerably more radiosensitive. "Radiation bronchitis", manifesting itself as a reddened hyperaemic airway mucosa and thickened bronchial secretions, following therapeutic external-beam radiation doses (50-60 Gy) applied to the lung has seldom been described. However, only a few articles have reported stenosis in proximal bronchi 615 months after external-beam radiation for lung cancer using 80 Gy doses [6], or tracheal stenosis after irradiation of tracheal cancers with doses ranging 50-70 Gy [7, 8]. No extrinsic compressions were noted.

In our observation, the right main-stem bronchial stenosis appeared to be extrinsic, being related to the presence of a right hilar and paratracheal mass disclosed $1 \mathrm{yr}$ after the completion of an external-beam radiation therapy. The absence of initial neoplastic mediastinal node involvement, the negativity of the repeated bronchial biopsies over a period as long as 42 months, and the results of the mediastinoscopy and of the successive thoracic CT led us to seriously consider the presence of post-radiotherapy sclerosing mediastinitis.

This case illustrates an unusual complication (bronchial stenosis) related to mediastinal fibrosis in the setting of thoracic radiotherapy, bearing in mind that the role of surgery cannot be excluded. To the best of our knowledge, this has not yet been reported. Potential factors for the onset of post-radiotherapy fibrosis have recently been discussed by Delanian et al. [9], emphazing the importance of the microvascular network supply. As our patient suffered from an advanced vascular disease, it is tempting to hypothesize that vascular derangement may have contributed to the enhancement of the mediastinal fibrotic process. It seems to us that, despite the lack of clinical reports on this hypothesis, this merits consideration as no other aetiological factors are suspected at the present time.

Acknowledgement: The authors are grateful to C. Hennequin, Hôpital Saint Louis, Paris, France, for his valuable advice. 


\section{References}

1. Mole TM, Glover J, Sheppard MN. Sclerosing mediastinitis: a report on 18 cases. Thorax 1995; 50: 280-283.

2. Whitcomb ME, Schwarz MI. Pleural effusion complicating intensive mediastinal radiation therapy. Am Rev Respir Dis 1971; 103: 100-107.

3. Rodriguez-Garcia JL, Fraille G, Moreno M, et al. Recurrent massive pleural effusion as a late complication of radiotherapy in Hodgkin's disease. Chest 1991; 100: 1165 1166.

4. Morrone N, Gama VL, Dourado AM, et al. Bilateral pleural effusion due to mediastinal fibrosis induced by radiotherapy. Chest 1993; 104: 1276-1278.

5. Rholl KS, Levitt RG, Glazer HS. Magnetic resonance imaging of fibrosing mediastinitis. Am $J$ Roentgenol 1984; 145: 255-259.

6. Hayakawa K, Mitsuhashi N, Saito Y, et al. Adverse chronic effects of high-dose irradiation on proximal bronchus in patients treated for bronchogenic carcinoma. $\mathrm{Br} \mathrm{J}$ Radiol 1993; 66: 477-479.

7. Baraka ME. Malignant tumours of the trachea. Ann $R$ Coll Surg Engl 1984; 66: 27-29.

8. Rostom AY, Morgan RL. Results of treating primary tumours of the trachea by irradiation. Thorax 1978; 33: 387-393.

9. Delanian S, Lefaix JL, Housset M. La fibrose iatrogénique en cancérologie (2è partie): principales étiologies et possibiltés thérapeutiques. Bull Cancer (Paris) 1993; 80: 202-212. 